\title{
In Vitro Study of Endotoxin Adsorption by a Polymyxin B-Immobilized Fiber Column
}

\author{
Chizuru Yamashita $^{a}$ Kazuhiro Moriyama $^{\mathrm{b}}$ Daisuke Hasegawa $^{\mathrm{a}}$ Yu Kato $^{\mathrm{a}}$ \\ Toshikazu Sakai $^{\text {a }}$ Takahiro Kawaji $^{a}$ Yasuyo Shimomura ${ }^{a}$ Yasuyoshi Kurimoto ${ }^{a}$ \\ Mariko Nagata ${ }^{a}$ Osamu Nishida ${ }^{a}$ \\ a Department of Anesthesiology and Critical Care Medicine, Fujita Health University School of Medicine, Toyoake, \\ Japan; 'baboratory for Immune Response and Regulatory Medicine, Fujita Health University School of Medicine, \\ Toyoake, Japan
}

\section{Keywords}

Polymyxin B-immobilized fiber · Endotoxin adsorption · Continuous endotoxin injection · Adsorption saturation . 24-h hemoperfusion

\section{Abstract \\ Background: Polymyxin B-immobilized fiber (PMX-F) col- umns are used as therapeutic interventions for septic shock. The clinical efficacy has been reported for 2-h applications, but their ability to adsorb endotoxin over longer treatments has not been fully elucidated. We hypothesized that PMX-F columns are capable of endotoxin removal for more than 2 h. Method: We designed closed circuits incorporating ei- ther a PMX-F column with an $8.5-\mathrm{mL}$ priming volume (PMX- $01 \mathrm{R}$ ) or a sham-control column, and used inactivated fetal bovine serum as the circulating perfusate. Endotoxin was continuously injected at a fixed rate for $24 \mathrm{~h}$, and perfusate endotoxin concentrations were measured at fixed time points. PMX-01R endotoxin adsorption was calculated from}

the difference in the endotoxin concentrations. Results: PMX-01R endotoxin adsorption increased continuously in a virtually linear manner. Conclusions: The PMX-01R column showed sustained endotoxin adsorption for at least $24 \mathrm{~h}$. This indicated that PMX-F columns would be capable of clinical endotoxin removal for $24 \mathrm{~h}$.

(c) 2018 The Author(s)

Published by S. Karger AG, Basel

\section{Introduction}

Polymyxin B-immobilized fiber (PMX-F) columns (Toraymyxin ${ }^{\circledR}$; Toray Industries, Tokyo, Japan) are cartridges containing polystyrene-derivative fibers with surfaces bearing covalently bonded polymyxin B [1]. The immobilized polymyxin B ionically binds with endotoxin lipid A, thereby enabling PMX-F columns to adsorb and eliminate endotoxin from blood. PMX-F columns were developed in Japan, where they have been marketed since 1994 as therapeutic interventions for septic shock. In an 
Fig. 1. Diagram of the in vitro experimental setup. The diagram shows the recirculation system employed for adsorption testing. A decrease in the endotoxin concentration is only observed when time-dependent adsorption occurs. LPS, lipopolysaccharide.

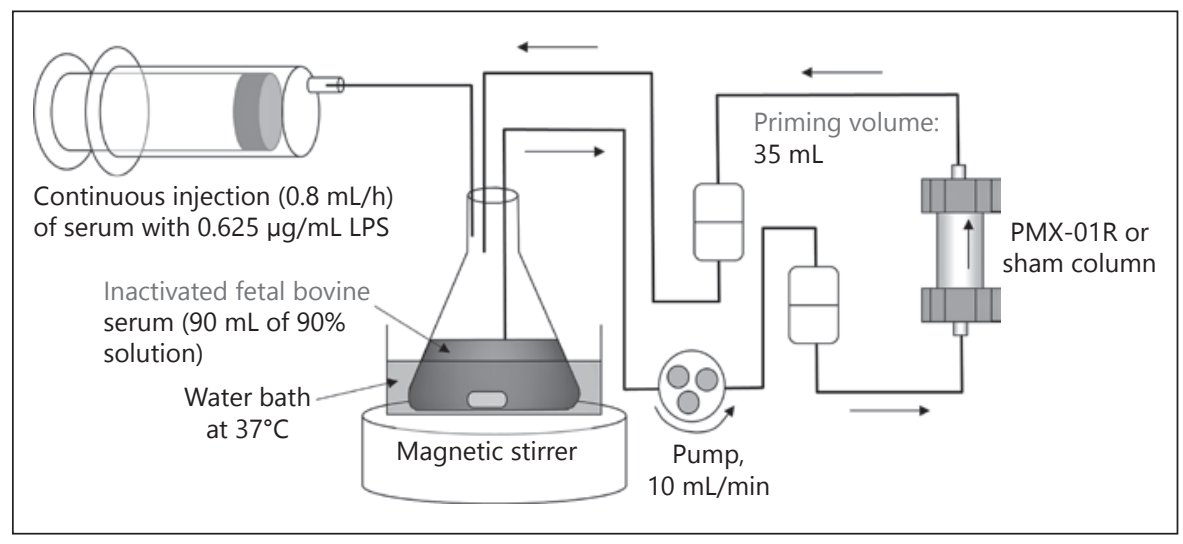

in vitro experiment conducted for Toray Industries, Sakai et al. [2] investigated PMX-F column endotoxin adsorption using endotoxin-spiked bovine serum in a closed circuit experimental setup. This study identified a reduction in the endotoxin level within $2 \mathrm{~h}$ of the start of treatment, and a constant endotoxin concentration thereafter. Based on these results, PMX-F column endotoxin adsorption was judged to be saturated within this time period, and PMX-F columns were marketed for a 2-h therapy duration. However, Sakai's experimental setup involved only a single introduction of endotoxin-spiked material, upon initiation of hemoperfusion. Although the endotoxin concentration plateaued, an adsorption limit can only be ascertained when the closed circuit endotoxin concentration increases after the addition of further endotoxinspiked material, and then remains at the increased level. Moreover, this experimental setup did not simulate the condition of sepsis patients that usually have persistent endotoxemia. A recent randomized control trial named the ABDO-MIX trial that applied this 2-h strategy failed to show a difference in mortality between the groups [3]. However, the study had potential problems that may have contributed to this observation [4]. Among the reasons, it was possible that short-term application of PMX-F columns was the reason for negative results in this trial. As a matter of fact, their ability to adsorb endotoxin over longer treatments has not been fully elucidated so far. Therefore, we hypothesized that the PMX-F column endotoxin adsorption capacity is retained for longer than $2 \mathrm{~h}$. This hypothesis is based on the results of numerous reports, which have indicated that PMX-F column hemoperfusion showed clinical efficacy for treatment durations exceeding this time period [5]. To test this hypothesis, we applied lipopolysaccharide (endotoxin) continually to closed circuits incorporating a sham-control or PMX-F column with an $8.5-\mathrm{mL}$ priming volume
(PMX-01R), and compared the rate of increase in the endotoxin concentration to investigate the duration of endotoxin adsorption capacity by PMX-F column.

\section{Materials and Methods}

The perfusate was prepared by mixing $135 \mathrm{~mL}$ of inactivated endotoxin-free fetal bovine serum (SA1720112, Sigma-Aldrich Corporation, Tokyo, Japan) with $15 \mathrm{~mL}$ injectable saline in a flask to prepare a $90 \%$ serum solution. Endotoxin-spiked serum was prepared by adding $36 \mathrm{~mL}$ fetal bovine serum to $4 \mathrm{~mL}$ endotoxin preparation (Escherichia coli 0111:B, L4391, Sigma-Aldrich Corporation, Tokyo, Japan), followed by adjustment of the endotoxin concentration to $0.625 \mu \mathrm{g} / \mathrm{mL}$ using injectable water. The endotoxin-spiked serum was loaded into a syringe. The in vitro experimental apparatus consisted of a PMX-01R column and a blood purification circuit for pediatric patients connected to a roller pump, and was washed with $0.5 \mathrm{~L}$ physiological saline solution. The priming volume of the PMX-01R column and the blood purification circuit was approximately $35 \mathrm{~mL}$. The physiological saline in the primed circuit was then gradually displaced by the perfusate from the flask, and the point at which the discarded volume reached $60 \mathrm{~mL}$ was regarded as $0 \mathrm{~h}$ (initiation of hemoperfusion). The volume of the perfusate in the flask at this point was $90 \mathrm{~mL}$. At this time, the perfusate in the flask was attached to the circuit incorporating the PMX-01R column, as shown in Figure 1, creating a closed circuit that returned the perfusate to the flask.

The perfusate was maintained in a thermoregulated bath at $37^{\circ} \mathrm{C}$; it was directly perfused (direct hemoperfusion) at a rate of $10 \mathrm{~mL} / \mathrm{min}$ for $24 \mathrm{~h}$, and drawn while being stirred with a magnetic stirrer. Endotoxin-spiked serum, which had been loaded into the syringe, was continuously injected into the flask at $0.8 \mathrm{~mL} / \mathrm{h}$ $(0.5 \mu \mathrm{g} / \mathrm{h})$ from $0 \mathrm{~h}$ onwards. The sham-control circuit was a closed circuit incorporating a tube instead of the PMX-01R column, and had the same capacity and volume of continuously injected endotoxin-spiked serum as the PMX-01R circuit. Samples of residual perfusate $(1 \mathrm{~mL})$ were collected from each circuit at $0.5,1,2,6,12$, 18 , and $24 \mathrm{~h}$ after the initiation of perfusion in Figure 2. Endotoxin concentration was measured by a toxinometer (MT-358; Wako Pure Chemical Industries, Ltd., Osaka, Japan) using a highly sensitive turbidimetric Limulus test method. Each collected sample was 
Fig. 2. Sampling points for endotoxin analysis. Samples were withdrawn from the flask at each collection point for endotoxin determination. The difference between the endotoxin concentration of the PMX-01R column and the sham-control circuit was multiplied by the solution volume to calculate the PMX-01R column endotoxin adsorption.

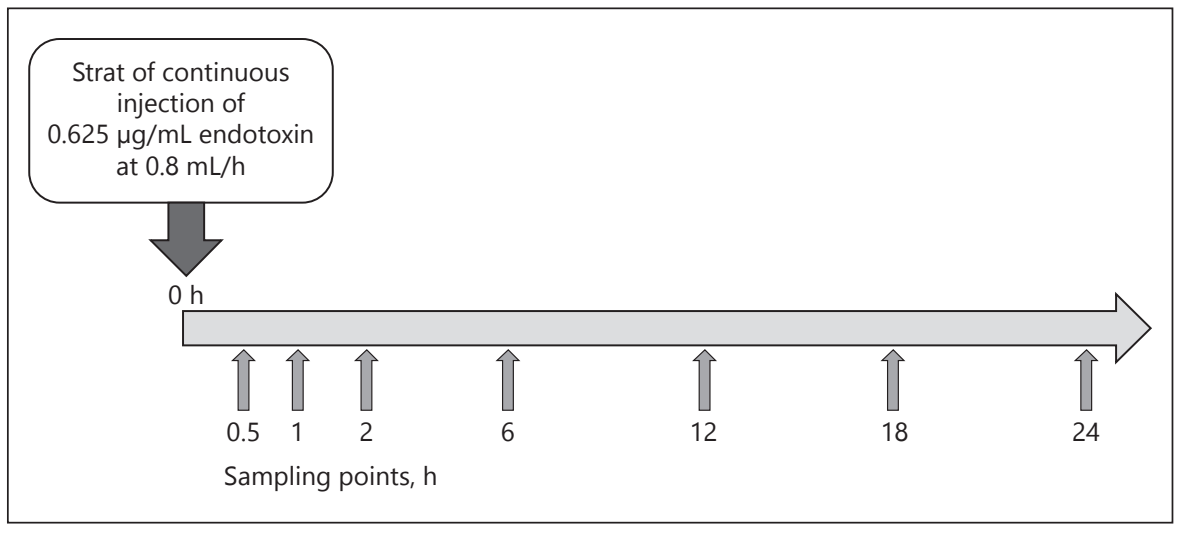

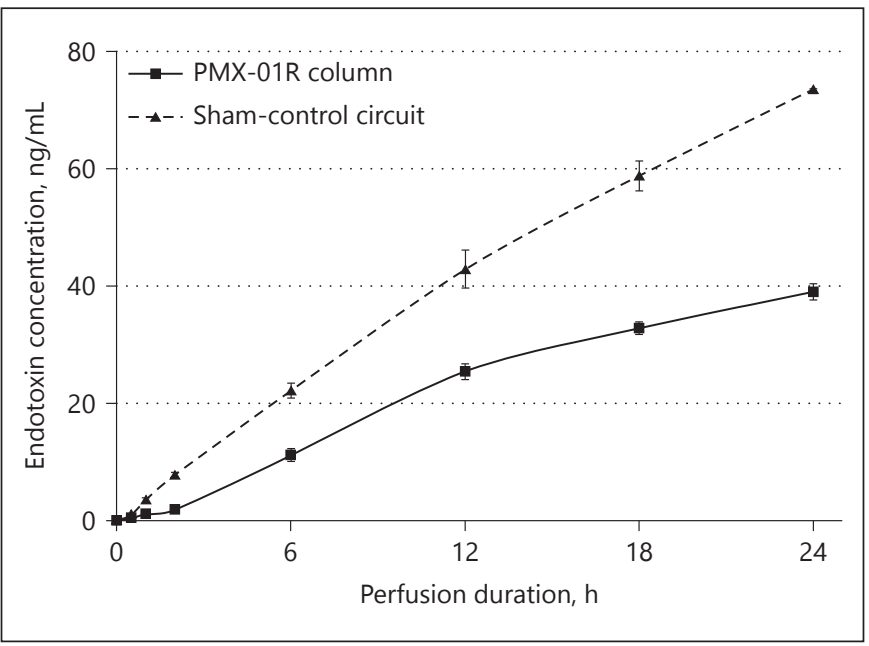

Fig. 3. Endotoxin concentration by time point. The hourly rate of increase in the endotoxin concentration slowed for both the PMX-01R column and the sham-control circuit after $12 \mathrm{~h}$; however, the difference in endotoxin concentration between the 2 circuits continued to widen through $24 \mathrm{~h}$.

diluted 10-fold with distilled injectable water, heat-treated for $10 \mathrm{~min}$ at $70^{\circ} \mathrm{C}$ to remove endotoxin inhibitors, and then immediately placed in an ice bath. The toxinometer measured the change in turbidity at a wavelength of $660 \mathrm{~nm}$, and the gelation time of the Limulus amebocyte lysate/endotoxin solution was assessed automatically. A calibration curve was constructed using serum samples with known endotoxin concentrations, and used to determine the sample endotoxin concentrations.

\section{PMX-01R Column Endotoxin Adsorption and Endotoxin}

Adsorption Ratio Calculation

The difference between the endotoxin concentration in the PMX-01R column circuit and that in the sham-control circuit was taken to indicate PMX-01R column endotoxin adsorption. The amount of adsorption at each measurement point was calculated by multiplying the difference between the PMX-01R column and sham-control circuits by the solution volume. The endotoxin adsorption ratio was calculated using the following equation:

Prolonged Endotoxin Adsorption by PMX Column
Endotoxin adsorption ratio $(\%)=($ control endotoxin concentration - PMX-01R endotoxin concentration)/control endotoxin concentration $\times 100$.

All results are expressed as the mean $\pm \mathrm{SD}$.

\section{Results}

Endotoxin concentrations in the PMX-01R column and sham-control circuits are shown for each time point in Figure 3. The sham-control circuit yielded endotoxin concentrations of $7.8 \pm 0.4,42.9 \pm 3.3$, and $73.5 \pm 0.1 \mathrm{ng} /$ $\mathrm{mL}$ after hemoperfusion for 2,12 , and $24 \mathrm{~h}$, respectively $(n=2)$. The PMX-F circuit yielded endotoxin concentrations of $1.9 \pm 0.1,25.4 \pm 1.3$, and $39.0 \pm 1.4 \mathrm{ng} / \mathrm{mL}$ at 2 , 12 , and $24 \mathrm{~h}$, respectively $(n=3)$. The hourly rate of endotoxin concentration increase slowed for both circuits from $12 \mathrm{~h}$; however, the difference in endotoxin concentrations in the 2 circuits had widened further by $24 \mathrm{~h}$.

The calculated amounts of PMX-01R column endotoxin adsorption are shown in Figure 4. These were $721.0 \pm 50.0,2,168.6 \pm 198.0$, and 4,728.8 $\pm 174.3 \mathrm{ng}$ at 2 , 12 , and $24 \mathrm{~h}$, respectively. The endotoxin adsorption ratio declined after $2 \mathrm{~h}$; however, PMX-01R column endotoxin adsorption showed a persistent and virtually linear increase from 2 to $24 \mathrm{~h}$.

Endotoxin adsorption ratios are shown by time point in Figure 5. The ratios at 2, 12, and $24 \mathrm{~h}$ were $75.4 \pm 1.5$, $39.7 \pm 1.2$, and $46.9 \pm 1.5 \%$, respectively.

\section{Discussion}

This study provides the first report of sustained PMX-F column endotoxin adsorption capacity over a 24 -h period of in vitro hemoperfusion. PMX-01R column endotoxin adsorption showed a linear increase through $24-\mathrm{h}$, when 


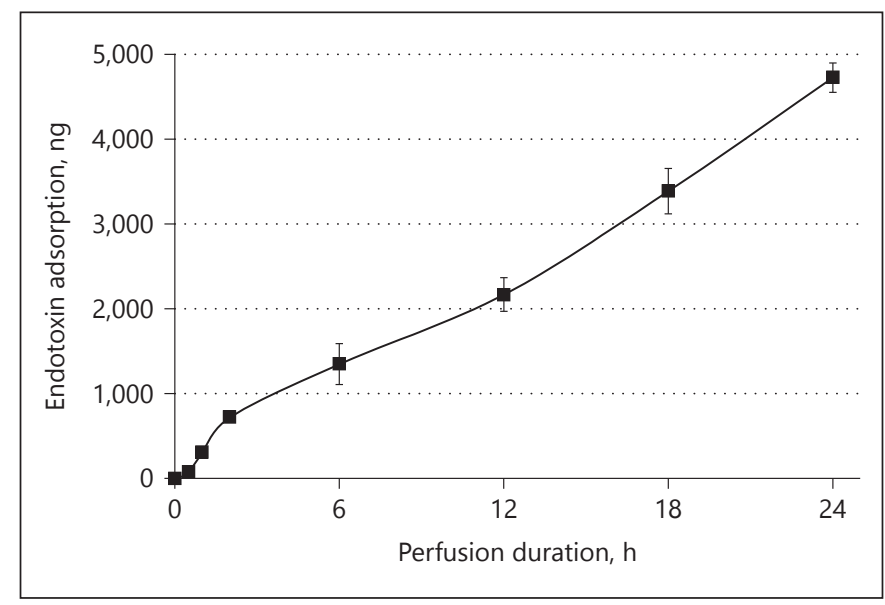

Fig. 4. PMX-01R column endotoxin adsorption by time point. The endotoxin adsorption rate decreased after approximately $2 \mathrm{~h}$, but total endotoxin adsorption showed a virtually linear increase from 2 to $24 \mathrm{~h}$.

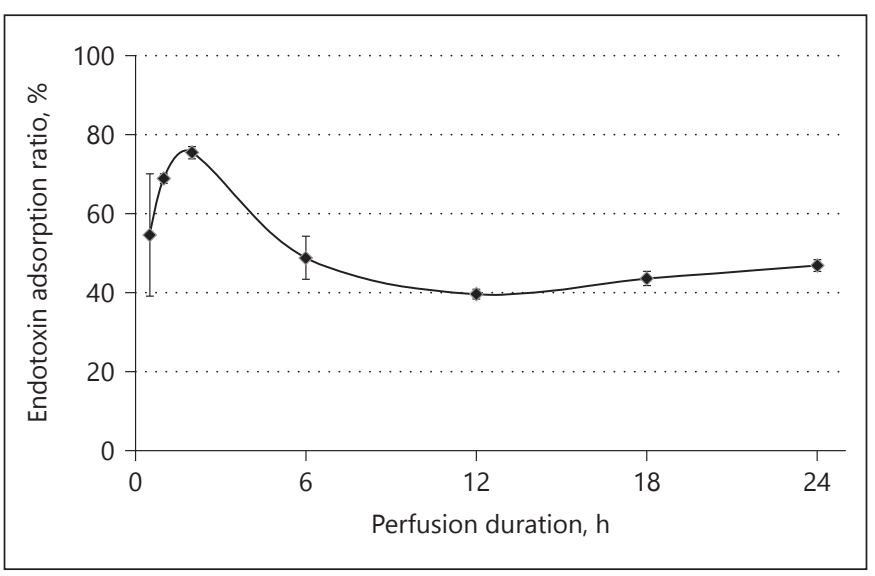

Fig. 5. Endotoxin adsorption ratio by time point. The endotoxin adsorption ratio showed high values of between 60 and $80 \%$ until $2 \mathrm{~h}$, and stabilized at around $40 \%$ thereafter.

it reached 4,730 ng. A stable endotoxin adsorption ratio was observed through 24 -h, with values consistently above $40 \%$. Until now, the study conducted by Sakai et al. [2] provided the only supporting data for PMX-F endotoxin adsorption capacity. That study reported on a 2-h perfusion period. However, Sakai's experimental setup did not demonstrate adsorption saturation because it involved a single introduction of endotoxin-spiked material, at the start of the perfusion period. Accordingly, the present study continuously injected endotoxin into the experimental setup. This resulted in the presence of a higher level of endotoxin, with a perfusate concentra- tion of $73.5 \pm 0.1 \mathrm{ng} / \mathrm{mL}$ in the sham-control circuit after $24 \mathrm{~h}$, as compared with an initial perfusate endotoxin concentration of $10 \mathrm{ng} / \mathrm{mL}$ in the Sakai's study. Even so, the PMX-01R column continued to adsorb endotoxin through $24 \mathrm{~h}$, when the saturation point had still not been reached. This finding indicated that the largely constant endotoxin concentration observed in Sakai's experimental setup represented an adsorption equilibrium, rather than saturation.

The PMX-01R column employed in the present study adsorbed a total of $4,730 \mathrm{ng}$ endotoxin in $24 \mathrm{~h}$. The PMX-01R column has a fiber loading of $4 \mathrm{~g}$, which is $1 / 14$ of that of the PMX-20R used by Sakai; this column has a $135-\mathrm{mL}$ priming volume when used for adult patients. Assuming that these columns have equivalent endotoxin adsorption capacities per gram of PMX-F, the extrapolated 24-h PMX-20R endotoxin adsorption is 66,220 ng. We calculated that only a fiftieth of the PMX-20R column adsorption capacity would be used within $2 \mathrm{~h}$ in an adult sepsis patient with an endotoxin concentration of $100 \mathrm{pg} /$ $\mathrm{mL}$ (although 2-figure $\mathrm{pg} / \mathrm{mL}$ levels or less are generally seen) and a blood flow of $100 \mathrm{~mL} / \mathrm{min}$, assuming that all of the 1,200 ng endotoxin passing through the column is adsorbed. Accordingly, we suggest that PMX-F columns have sufficient capacity to adsorb endotoxin throughout 24-h hemoperfusions, without reaching saturation.

High endotoxin adsorption ratios were observed, ranging between 60 and $80 \%$ for 2 -h hemoperfusions, and reducing slightly to approximately $40 \%$ thereafter. This phenomenon may indicate a high rate of endotoxin adsorption at the start of hemoperfusion, when the fibers were free of protein adhesion, and a lower subsequent rate of endotoxin adsorption as proteins adhered to the fibers.

The findings of the present study were consistent with our previous observation that hemoperfusion (mean therapy duration: $15.9 \mathrm{~h}$ ) with a PMX-F column showed sustained clinical efficacy for hemodynamics and pulmonary oxygenation [5]. In addition, a PMX-F column was reported to show sustained adsorption during 24-h hemoperfusion therapy in septic shock patients, with endotoxin concentrations measured upon entry to and exit from the column [6].

This study had a number of limitations. Firstly, protein inactivation was required for endotoxin measurement, and we did not use whole blood. However, we considered that this experimental setup was adequate enough for determination of the PMX-01R column endotoxin adsorption capacity. Secondly, the rate of increase in the endotoxin concentration slowed for both the sham-control 
circuit and the PMX-01R column from around the 12-h time point. The reason for this remains unclear, although we suspect the involvement of time-dependent changes such as endotoxin degradation. However, the same trend was present in both circuits and we consider that the comparison was valid through $24 \mathrm{~h}$. The third limitation concerned the limit of endotoxin adsorption by the PMX-01R column; this was not elucidated because the column did not reach saturation during this 24-h experiment. Therefore, adsorption saturation time of PMX and adsorption amount should be examined.

Further, the efficacy of this strategy ultimately needs to be confirmed in clinical settings. Randomized clinical studies of longer duration PMX-F column hemoperfusion are warranted in patients with septic shock, in order to investigate the clinical efficacy of this therapy over prolonged time periods.

\section{Conclusions}

The PMX-01R column was found to have a sustained endotoxin adsorption capacity throughout a 24 -h hemoperfusion in the closed circuit experimental setup employed in this study. This finding and the blood endotoxin concentrations typically observed in patients with septic shock indicated that PMX-F columns are capable of clinical endotoxin removal for 24-h hemoperfusions. These findings warrant further in vivo investigation of longer duration PMX-F column hemoperfusion, as well as clinical evaluation in patients with sepsis.

\section{Disclosure Statement}

The authors declare no conflict of interest.

\section{References}

1 Shoji H, Tani T, Hanasawa K, Kodama M: Extracorporeal endotoxin removal by polymyxin B immobilized fiber cartridge: designing and antiendotoxin efficacy in the clinical application. Ther Apher 1998;2:3-12.

2 Sakai Y, Shoji H, Kobayashi T, Terada R, Sugaya $\mathrm{H}$, Murakami M, Moriyama K, Minaga M, Kunitomo T, Takeyama T: New extracorporeal blood purification devices for critical care medicine under development. Ther Plasmapheresis 1993;12:837-842.
3 Payen DM, Guilhot J, Launey Y, Lukaszewicz AC, Kaaki M, Veber B, Pottecher J, JoannesBoyau O, Martin-Lefevre L, Jabaudon M, Mimoz O, Coudroy R, Ferrandière M, Kipnis E, Vela C, Chevallier S, Mallat J, Robert R; ABDOMIX Group: Early use of polymyxin B hemoperfusion in patients with septic shock due to peritonitis: a multicenter randomized control trial. Intensive Care Med 2015;41:975-84.

4 Antonelli M, Ronco C: Polymyxin B hemoperfusion in sepsis: growing body of evidence and occasional conflicting results. Blood $\mathrm{Pu}$ rif 2015;39:I-II.
5 Yamashita C, Hara Y, Kuriyama N, Nakamura T, Nishida O: Clinical effects of a longer duration of polymyxin B-immobilized fiber column direct hemoperfusion therapy for severe sepsis and septic shock. Ther Apher Dial 2015;19:316-323.

6 Mitaka C, Fujiwara N, Yamamoto M, Toyofuku T, Haraguchi G, Tomita M. Polymyxin Bimmobilized fiber column hemoperfusion removes endotoxin throughout a 24-hour treatment period. J Crit Care 2014;29:728-732. 